\title{
Visceral adiposity index correlation with Rotterdam criteria in patients with polycystic ovary syndrome
}

\author{
Mohamed M. Aboelnaga ${ }^{1}$, H. Elshahawy ${ }^{2}$
}

${ }^{1}$ Lecturer of Endocrinology, Faculty of Medicine, Mansoura University, Mansoura, Egypt
${ }^{2}$ Lecturer of Clinical Pathology, Faculty of Medicine, Mansoura University, Mansoura, Egypt

Received: 06 June 2016

Accepted: 01 July 2016

*Correspondence:

Dr. Mohamed M. Aboelnaga,

E-mail: dr.mhd.endocrine@gmail.com

Copyright: $\odot$ the author(s), publisher and licensee Medip Academy. This is an open-access article distributed under the terms of the Creative Commons Attribution Non-Commercial License, which permits unrestricted non-commercial use, distribution, and reproduction in any medium, provided the original work is properly cited.

\begin{abstract}
Background: The present research explores the correlation of visceral obesity index with Rotterdam criteria (hyperandrogenism and/or hyperandrogenemia, oligomenorrhea and Ultrasound polycystic ovarian morphology) among Egyptian polycystic ovary syndrome patients.

Methods: We enrolled one hundred female patients with polycystic ovary syndrome with age ranged 18-44 years (mean age 26.83 \pm 6.092 years).

Results: VAI very strongly correlated with waist circumference, TG and HDL-c, also moderately correlated with systolic B.P, BMI, HOMA-IR and insulin levels, also we found only insulin, menstrual cycles per year number and FGS were significant predictors of hyperandrogenemia in PCOS patients. Only Ultrasound polycystic ovarian morphology was a significant risk factor for oligomenorrhea in PCOS patients in logistic regression analysis. VAI, TT and Farman gallawy score can significantly predict number of the menstrual cycles per year number $(p<0.05)$. In logistic regression analysis, only oligomenorrhea was a significant independent risk factor for PCOM ( $<<0.05)$. In addition, only VAI was a significant independent $(\mathrm{p}<0.05)$ risk factor for metabolic syndrome.

Conclusions: VAI was an independent significant predictor for metabolic syndrome in patients with PCOS and a good marker of cardiometabolic risk in PCOS patients. In addition, VAI was significant predictors of annual menusteral cycle but not testosterone levels or polycystic ovarian morphology in PCOS. This study confirms the value of VAI in identification of patient with risk for metabolic syndrome and cardiometabolic risk in PCOS patients, but not found a role for VAI in diagnosis of PCOS.
\end{abstract}

Keywords: Hyperandrogenism, Oligomenorrhea, PCOS, VAI

\section{INTRODUCTION}

Polycystic ovary syndrome (PCOS) is one of the most common endocrine disorders of women at reproductive age and the major cause of anovulatory infertility. ${ }^{1}$ Chereau first described it as the change of ovarian morphology in $1844 .^{2}$ PCOS is a disease with high heterogeneity, and its clinical features mainly include menstrual disorder, secondary amenorrhea, serum hormone abnormality, hairiness, acne, obesity, and infertility. ${ }^{3}$
Recently, the use of visceral adiposity index (VAI), a sex-specific mathematical model that uses both anthropometric [body mass index (BMI) and waist circumference (WC)] and functional [triglycerides (TG) and high-density lipoprotein cholesterol (HDL)] parameters, has been suggested as an index of applicable index for the evaluation of visceral fat dysfunction. ${ }^{4}$

In the last years, the VAI has been reported to express cardiometabolic risk and possible adipose tissue dysfunction (ATD) was identified in populations with 
metabolic risks such as the general population with obesity, post-menopausal women with non-alcoholic fatty liver disease in patients with acromegaly, the VAI appears to be associated with disease activity, adiponectin levels, insulin sensitivity and secretion, and women with PCOS. $^{10}$

Recent studies evaluated VAI in women with PCOS and reported that VAI correlated with insulin resistance, differentiate the "metabolically healthy polycystic ovary syndrome" from the "metabolically unhealthy polycystic ovary syndrome" Also VAI replace visceral CT scan as a marker for visceral adiposity. ${ }^{11-13} \mathrm{VAI}$ is considered a predictor of clinical severity and therapeutic outcome of PCOS. ${ }^{14}$ And a predictor of diabetes risk in women with polycystic ovary syndrome. ${ }^{15}$

PCOS is characterized by hyperandrogenemia, Insulin resistance, chronic anovulation, and polycystic ovary morphology. ${ }^{16}$ Hyperandrogenemia is the biochemical hallmark of PCOS. ${ }^{17}$ Previous studies hypothesized that hyperinsulinemia causes hyperandrogenemia and anovulation in PCOS. ${ }^{18}$ On the other hand other studies, since many normal-weight PCOS patients are not insulin resistant, the hypothesis that hyperandrogenism per se leads to abdominal visceral adiposity, insulin resistance and hyperinsulinemia has been postulated by many authors. ${ }^{17}$

Visceral adiposity index is a marker of ATD and correlated with insulin resistance in PCOS. ${ }^{13}$ Relation between VAI with hyperandrogenism and anovulation in PCOS is unclear. So the evaluation of this relationship will be valuable in order to evaluate of VAI as marker of the main criteria of polycystic ovary syndrome, not ATD alone. The aim of this study was to evaluate the correlation of visceral obesity index with Rotterdam criteria (hyperandrogenism and/or hyperandrogenemia, oligomenorrhea and ultrasound polycystic ovarian morphology) among Egyptian polycystic ovary syndrome patients.

\section{METHODS}

We enrolled one hundred female patients with polycystic ovary syndrome with age ranged 18-44 years (mean age $26.83 \pm 6.092$ years). This study was conducted at specialized medical hospital and Mansoura university hospital, in Egypt, from July 2012 to December 2015.

Informed consent obtained from all participants. Inclusion criteria were as follows: 18 to 45 female patients with polycystic ovary syndrome (diagnosed according to the Rotterdam criteria which require the presence of two of three: oligomenorrhea (less than 6-9 menses per year), clinical hyperandrogenism (hirsutism defined as a Ferriman-Gallwey (FG) score $\geq 8$, acne) or biochemical hyperandrogenism (serum total testosterone [TT] $>0.8 \mathrm{ng} / \mathrm{mL}$ upper limit of the present laboratory) and polycystic ovary appearance on ultrasonography $(\geq 12$ follicles measuring 2-9 $\mathrm{mm}$ in at least one ovary and/or increased ovarian volume, $>10 \mathrm{~mL}$ ), with adequate hepatic and renal function, and absence of malignancies, metabolic or any osseous diseases. ${ }^{19,20}$

Exclusion criteria involved hepatic or renal disease, abnormal albumin levels, patients with autoimmune disease and endocrine disorder including type 2 diabetes (abnormal thyroid function, hypercrtolism, hyperprolactinemia, abnormal calcium\}, hypertensive patient and cardiac disease.

BMI was calculated from body weight and height by the standard formula: $\mathrm{BMI}=$ (weight in $\mathrm{kg} /$ height in $\mathrm{m}^{2}$ ). Waist circumference was estimated as the smallest circumference at the level of the umbilicus. VAI was calculated using the proposed formula: $\mathrm{VAI}=\mathrm{WC} / 36.58$ $+($ one $.89 \times \mathrm{BMI}) \times \mathrm{TG} / 0.81 \times 1.52 / \mathrm{HDL}^{4}$

Laboratory evaluation included; laboratory evaluation included; basal prolactin $(\mathrm{ng} / \mathrm{ml})$, total testosterone (ng/ml), FBS ( $\mathrm{mg} / \mathrm{dl})$, insulin (mIu/ml), Hb (g/dl), Hct\%, WBC (X109/L), PLT (X109/L), uric acid, SGOT, SGPT, HDL-c, LDL-c $(\mathrm{mg} / \mathrm{dl})$ and TG $(\mathrm{mg} / \mathrm{dl})$ during the follicular phase. All blood samples were obtained at 09:00 hours in the morning after overnight fasting. Homeostasis model assessment for insulin resistance (HOMA-IR) was calculated using the standard formula: fasting glucose $(\mathrm{mm}) /$ fasting insulin $(\mathrm{mIU} / \mathrm{ml}) / 22.5$. Insulin sensitivity was estimated indirectly using basal insulin and glucose values to calculate the HOMA-IR [glycemia $(\mathrm{mg} / \mathrm{dl}) \times$ insulinemia $(\mathrm{mU} / \mathrm{ml}) / 405 .^{22}$

All data were analyzed using the SPSS statistical version 22.0. Pearson correlation was used to assess the correlation between VAI, total testosterone and number of menstrual cycle per year with other parameters, significant correlation was entered in multiple regression analysis for determination of independent predictors. An independent $t$ test was used for comparison of continuous variables. Categorical data were analyzed by the Pearson Chi-square. A multivariate analyses model (enter method) was used to examine the relationship between polycystic ovarian morphology and metabolic syndrome with other significant parameters. $\mathrm{P}$ values less than 0.05 were considered significant.

\section{RESULTS}

We enrolled one hundred female patients with polycystic ovary syndrome from July 2012 to December 2015 with mean age $26.83 \pm 6.09$ years (ranged from 18 year to 44 ). Details of patient's characteristics were presented in Table 1.

\section{VAI correlation and independent predictors}

In this study we found visceral adiposity index had statistically significant correlation ( $\mathrm{p}<0.05)$ with anthropometric parameter (waist circumference, BM and 
Weight), clinical parameter (systolic B.P, diastolic B.P, menstrual cycles per years and Farman Gallwey score), biochemical (HDL-c and triglyceride), and hormonal parameter total testosterone, insulin and HOMA IR.

Table 1: patient's characteristics.

\begin{tabular}{|lll|}
\hline Age & Mean & $\begin{array}{l}\text { Standard } \\
\text { deviation }\end{array}$ \\
\hline Weight & 26.83 & 6.092 \\
\hline Height & 82.8300 & 15.01787 \\
\hline BMI & 1.5978 & 0.03963 \\
\hline Waist circumference & 32.5057 & 6.27400 \\
\hline VAI & 115.44 & 16.468 \\
\hline Systolic B.P & 3.7736 & 0.99776 \\
\hline Diastolic B.P & 132.7500 & 14.07726 \\
\hline
\end{tabular}

\begin{tabular}{|lll|}
\hline Menstrual cycles per years & 7.55 & 1.956 \\
\hline Farman Gallawy score & 13.94 & 3.598 \\
\hline Total cholesterol & 229.0500 & 30.18775 \\
\hline LDL-c & 142.9900 & 27.61458 \\
\hline HDL-c & 48.7900 & 7.71630 \\
\hline Triglyceride & 186.3500 & 39.88959 \\
\hline FBS & 94.76 & 9.322 \\
\hline Total testosterone & 99.3000 & 14.62632 \\
\hline Prolactin & 17.6670 & 3.08620 \\
\hline TSH & 2.4444 & 0.85217 \\
\hline Insulin & 12.4593 & 5.78178 \\
\hline HOMA & 2.9290 & 1.44578 \\
\hline Metabolic syndrome & $59(59 \%)$ & \\
\hline Oligomenorrhoea & $66(66 \%)$ & \\
\hline $\begin{array}{l}\text { Ultrasound polycystic } \\
\text { ovarian morphology }\end{array}$ & $87(87 \%)$ & \\
\hline
\end{tabular}

Table 2: Pearson correlations between VAI with laboratory and clinical parameters and regression analysis (stepwise method) with significant correlated parameters for determination of VAI predictors PCOS patients.

\begin{tabular}{|c|c|c|c|c|c|}
\hline & $\mathbf{r}$ & P value & B & $\beta$ & P value \\
\hline Age & 0.034 & 0.737 & & & \\
\hline Waist circumference & 0.417 & $<0.001 *$ & 0.032 & 0.534 & $<0.001^{*}$ \\
\hline BMI & 0.274 & $0.006^{*}$ & -0.072 & -0.453 & $<0.001^{*}$ \\
\hline Weight & 0.275 & $0.003^{*}$ & \multicolumn{3}{|c|}{ Excluded in stepwise regression } \\
\hline Height & -0.091 & 0.366 & & & \\
\hline Systolic BP & 0.204 & $0.046^{*}$ & \multicolumn{3}{|c|}{ Excluded in stepwise regression } \\
\hline Diastolic BP & 0.135 & 0.179 & & & \\
\hline Menstrual cycles per years & -0.313 & $0.002 *$ & -0.024 & -0.047 & $0.013 *$ \\
\hline Farman Gallawy score & 0.240 & $0.013^{*}$ & \multicolumn{3}{|c|}{ Excluded in stepwise regression } \\
\hline Total cholesterol & -0.001 & 0.994 & & & \\
\hline HDL-c & -0.561 & $<0.001 *$ & 2.736 & -0.547 & $<0.001^{*}$ \\
\hline LDL-c & -0.056 & 0.583 & & & \\
\hline Triglyceride & 0.723 & $<0.001 *$ & 1.649 & 0.745 & $<0.001 *$ \\
\hline Total testosterone & 0.248 & $0.016 *$ & 0.003 & 0.041 & 0.035* \\
\hline Prolactin & 0.101 & 0.315 & & & \\
\hline TSH & -0.016 & 0.873 & & & \\
\hline FBS & -0.103 & 0.310 & & & \\
\hline Insulin & 0.240 & $0.016 *$ & Exclud & epwise $r$ & \\
\hline HOMA & 0.214 & $0.033 *$ & Exclud & epwise $r$ & \\
\hline
\end{tabular}

*Correlation is significant at the 0.05 level (2-tailed).

However, in regression analysis by stepwise method to eliminate effect of collinearity, we found only total testosterone, menstrual cycles per year number and the lipid parameters of the index (HDL-c and Triglyceride) and anthropometric parameters Waist circumference, BMI as shown in Table 2.

\section{Hyperandrogenemia}

In present study, we found total testosterone had statistically significant correlation $(\mathrm{P}<0.05)$ with anthropometric parameter (waist circumference, BM and weight), clinical parameter (systolic B.P, diastolic B.P, menstrual cycles per years and Farman Gallwey score), VAI, HDL-c, insulin and HOMA IR.

However, in regression analysis, we found only insulin, menstrual cycles per year and FGS were significant predictors of hyperandrogenemia in polycystic ovarian syndrome as shown in Table 3.

\section{Oligomenorrhea}

Only Ultrasound polycystic ovarian morphology was a significant risk factor for Oligomenorrhea in PCOS patients in logistic regression analysis $(p<0.05)$ as presented in Table 4. 
Table 3: Pearson correlation and multiple regression (enter method) analysis between total testosterone levels with other statistically significant correlated factors.

\begin{tabular}{|llllcc|}
\hline & r & P value & B & $\beta$ & P value \\
\hline Waist circumference & 0.274 & $0.006^{*}$ & 0.111 & 0.125 & 0.374 \\
\hline BMI & 0.258 & $0.005^{*}$ & \multicolumn{2}{c}{ Excluded because of collinearity with weight } \\
\hline Weigh & 0.298 & $0.04^{*}$ & 0.016 & 0.016 & 0.902 \\
\hline VAI & 0.248 & $0.013^{*}$ & -1.560 & -0.106 & 0.339 \\
\hline Systolic B.P & 0.232 & $0.019^{*}$ & 0.038 & 0.036 & 0.699 \\
\hline Diastolic B.P & 0.207 & $0.039^{*}$ & Excluded because of collinearity with systolic B.P \\
\hline Cycles number/year & -0.413 & $0.001^{*}$ & -1.934 & -0.259 & $0.005^{*}$ \\
\hline Farman Gallawy score & 0.473 & $<0.001^{*}$ & 1.581 & 0.389 & $<0.001^{*}$ \\
\hline HDL-c & -0.231 & $0.021^{*}$ & -8.046 & -0.110 & 0.267 \\
\hline Insulin & 0.392 & $<0.001^{*}$ & 0.575 & 0.227 & $0.023^{*}$ \\
\hline HOMA & 0.380 & $0.001^{*}$ & Excluded because of collinearity with Insulin \\
\hline
\end{tabular}

*significant at the 0.05 level.

Table 4: Logistic regression analyses (enter method) for predictors of oligo-ovulation in PCOS patients.

\begin{tabular}{|llllllll|} 
& B & S.E. & Wald & Signature & Odd ratio & \multicolumn{2}{c|}{ L5\% C.I for OR } \\
\hline Metabolic syndrome & -0.863 & 0.594 & 2.110 & 0.146 & 0.422 & 0.132 & 1.352 \\
\hline VAI & -0.026 & 0.317 & 0.007 & 0.935 & 0.974 & 0.523 & 1.815 \\
\hline HOMA & 0.116 & 0.232 & 0.251 & 0.616 & 1.123 & 0.713 & 1.771 \\
\hline TT & 0.015 & 0.019 & 0.641 & 0.424 & 1.015 & 0.978 & 1.054 \\
\hline PCOM & -2.757 & 0.775 & 12.651 & $<0.001 *$ & 0.063 & 0.014 & 0.290 \\
\hline
\end{tabular}

*Significant at the 0.05 level.

Table 5: Pearson correlation and multiple regression (enter method) analysis between number of menusteral cycle per year with other statistically significant correlated factors.

\begin{tabular}{|lllll|}
\hline & r & P value & B & $\beta$ \\
\hline VAI & 0.313 & $0.002^{*}$ & -0.387 & -0.197 \\
\hline Systolic B.P & 0.248 & $0.013^{*}$ & -0.017 & -0.123 \\
\hline Diastolic B.P & 0.212 & $0.034^{*}$ & Excluded because of Collinearity with Systolic B.P \\
\hline TT & -0.413 & $0.001^{*}$ & -0.042 & -0.313 \\
\hline Farman Gallawy score & 0.219 & $-0.005^{*}$ & -0.009 & 0.929 \\
\hline Insulin & 0.246 & $0.013^{*}$ & -0.015 & -0.046 \\
\hline HOMA & 0.225 & $0.025^{*}$ & Excluded because of Collinearity with Insulin \\
\hline
\end{tabular}

*significant at the 0.05 level.

Table 6: Logistic regression analyses (enter method) for predictors of ultrasound polycystic ovarian morphology (PCOM).

\begin{tabular}{|llllllll|} 
& B & S.E. & Wald & Signature & Odd ratio & \multicolumn{2}{c|}{ 95\% C.I for OR } \\
\hline Total testosterone & 0.029 & 0.029 & 1.040 & 0.308 & 1.030 & 0.973 & Upper \\
\hline Oligo-ovulation & -2.507 & 0.704 & 12.693 & $<0.001 *$ & 0.082 & 0.021 & 0.324 \\
\hline Diastolic B.P & 0.007 & 0.064 & 0.012 & 0.913 & 1.007 & 0.889 & 1.141 \\
\hline Systolic B.P & 0.030 & 0.043 & 0.483 & 0.487 & 1.030 & 0.947 & 1.121 \\
\hline
\end{tabular}

*Significant at the 0.05 level. 
However, by linear regression analysis VAI, TT and Farman Gallwey score can significantly predict number of the menstrual cycles per year number $(\mathrm{p}<0.05)$. Also in Pearson correlation, we found menstrual cycles per year number had statistically significant correlation with VAI, systolic and Diastolic B.P, Insulin, HOMA IR, TT and Farman Gallawy score as described in Table 5.

Ultrasound polycystic ovarian morphology (PCOM)
In logistic regression analysis, only oligomenorrhea was a significant independent risk factor for PCOM $(p<0.05)$ as described in Table 6.

\section{Metabolic syndrome}

In logistic regression analysis for determination of risk factor of metabolic syndrome in PCOS patients, only VAI was a significant independent $(\mathrm{p}<0.05)$ risk factor for metabolic syndrome (Table 7).

Table 7: Logistic regression analyses (enter method) for metabolic syndrome risk factors in PCOS patients.

\begin{tabular}{|llllllll|} 
& B & S.E. & Wald & Signature & Odd ratio & \multicolumn{2}{c|}{ L5\% C.I for OR } \\
\hline Waist circumference & 0.011 & 0.027 & 0.166 & 0.684 & 1.011 & 0.959 & 1.066 \\
\hline VAI & 1.395 & 0.376 & 13.737 & $<0.001 *$ & 4.033 & 1.929 & 8.433 \\
\hline Systolic B.P & 0.029 & 0.034 & 0.728 & 0.394 & 1.029 & 0.963 & 1.100 \\
\hline Diastolic B.P & 0.003 & 0.057 & 0.003 & 0.954 & 1.003 & 0.897 & 1.123 \\
\hline Farman Gallawy score & 0.103 & 0.085 & 1.464 & 0.226 & 1.109 & 0.938 & 1.311 \\
\hline Total testosterone & 0.001 & 0.023 & 0.002 & 0.964 & 1.001 & 0.957 & 1.047 \\
\hline HOMA & 0.119 & 0.226 & 0.279 & 0.598 & 1.127 & 0.723 & 1.755 \\
\hline BMI $\geq 30 \mathrm{Kg} / \mathrm{m}^{2}$ & 0.634 & 0.944 & 0.451 & 0.502 & 1.886 & 0.296 & 12.005 \\
\hline oligoovulation & -0.526 & 0.545 & 0.933 & 0.334 & 0.591 & 0.203 & 1.718 \\
\hline
\end{tabular}

*Significant at the 0.05 level.

\section{DISCUSSION}

In the present study, we investigated the relation of the component of PCOS according Rotterdam criteria oligoamenorrhea, clinical hyperandrogenism or hyperandrogenemia and US polycystic ovarian morphology with visceral adiposity index (VAI) as a marker metabolic dysfunction of visceral obesity.

In this study we found total testosterone correlated with VAI with statistically significance in agreement Andreoulakis et al results, but previous study depend only on Pearson correlation only for their conclusion which not enough to conclude a role for VAI in determination of TT levels in PCOS patients. ${ }^{17}$ In regression analysis, we found VAI was non-significant predictor of total testosterone levels in polycystic ovarian disease patients.

By contrast, we found TT level was a significant predictor for VAI in PCOS which in agreement with $\mathrm{Oh}$ et al results. ${ }^{13}$ These finding indicated that hyperandrogenemia leads to visceral obesity and adipose tissue dysfunction cystic ovarian disease patients.

Hyperandrogenemia is the biochemical hallmark of PCOS. $^{21}$ The mechanisms explained the association between visceral obesity and hyperandrogenism was controversial. Some studies suggested insulin resistance, studies suggested that insulin also contributes to hyperandrogenemia and anovulation in affected women through a number of distinct molecular mechanisms involved insulin receptor and post receptor defect. ${ }^{22,23}$ Other studies suggested that inappropriate gonadotropin secretion and exaggerated ovarian response to gonadotropin, which one of the most characteristic biochemical abnormalities in PCOS is involved in hyperandrogenemia and insulin resistance pathogenesis. ${ }^{24}$ In current study insulin was a significant predictor for TT levels which indicated a pivotal role for insulin resistance in hyperandrogenemia pathogenesis. Unfortunately pituitary and hypothalamic role not evaluated in our study.

On the other hand, other studies reported that hyperandrogenemia per se can leads to abdominal visceral adiposity, insulin resistance and hyperinsulinemia, which had been, reported previously. ${ }^{25}$

In the same veins, we found number of menstrual cycle per year significantly correlated with VAI. In addition, in regression analysis we found VAI was significant independent predictors for number of menstrual cycles per year in women with polycystic ovarian syndrome. These findings were in agreement with other studies [10.17] reported that higher VAI associated with Oligomenorrhea in PCO women, By contrast another 
study reported obesity does not appear to have an important effect on menstrual cycle pattern in PCOS. ${ }^{26}$

Multiple studies have found associations between overweight or obesity and irregular menstruation in women without PCOS. ${ }^{27,28}$ The risks for oligomenorrhea and amenorrhea increase 2-fold with each increase in obesity grade. $^{29}$ Adipose tissue appears to exert its greatest effects on reproductive cyclicity via complex mechanisms involving hyperandrogenism and hyperinsulinemia. ${ }^{30}$

This controversy may explain by multiple reasons, firstly the subjective nature of cycle irregularity, which can easily over or underestimated. Secondly genetic and ethnicity differences between studies. Thirdly diagnosis of PCOS vary according cafeteria used for diagnosis. The Rotterdam criteria did not require irregular menses or ovulatory dysfunction for diagnosis citing that women with regular menstrual cycles could be considered to have PCOS in the presence of PCO and hyperandrogenemia or hyperandrogenism. ${ }^{19}$ While androgen excess society (Aes) excludes the diagnosis of PCOS in women with regular menses and subclinical ovulatory dysfunction. ${ }^{31}$ Subclinical ovulatory dysfunction can occur in women with regular menstrual bleeding. ${ }^{32}$ Fourthly, high rates of amenorrhea and menstrual irregularity and the prevalence may be associated with certain stresses, which not included in most of studies. ${ }^{33}$

In the same veins, we found women with polycystic ovarian US morphology (PCOM) had statistically significant higher VAI than those without PCOM appearance by US. These findings were similar with other studies in PCOS women, but in logistic regression analysis we found visceral obesity was none a significant risk factor for US polycystic ovarian morphology. ${ }^{34}$

The morphological features of the ovaries in women with polycystic ovarian syndrome include enlarged ovary size, multiple small follicles of similar size, increased ovarian stromal volume, and echogenicity, peripheral distribution of the follicles, and higher stromal blood flow. Ultrasound polycystic ovarian morphology (PCOM) has been recognized as a component of PCOS diagnosis. The inclusion of PCOM sparked a controversy as it broadens the population of women who meet the criteria for PCOS and allows for the creation of two phenotypically different patient populations who previously would have been excluded. ${ }^{35}$ gonadotropin secretion and action as important primary defects in disease pathogenesis and some gene regions regulating $\mathrm{FSH}$ and $\mathrm{LH}$ secretion dysfunction, suggesting genetic susceptibility in PCOS pathogenesis and morphology. ${ }^{36}$

Also In this study we found VAI was an independent significant predictors for metabolic syndrome in patients with PCOS. These findings is contestant with other recent studies reported VAI as a predictor for metabolic syndrome in PCOS patients. ${ }^{34,37}$ In the present study, VAI was very strongly correlated with waist circumference, , TG and HDL-c , also moderately correlated with systolic B.P ,BMI, HOMA-IR and insulin levels. Therefore, in the present study it is not surprising that VAI had a good value in predicting Met Syndrome in PCOS women and a good marker of cardiometabolic risk in PCOS patients.

The relation between metabolic syndrome and PCOS phenotype was controversial, some studies in turkey and Iran reported high prevalence in all phenotypes while studies in Latin population found incidence of Met Syndrome in all phenotypes except type A was not different from that in non-PCOS women. ${ }^{34,38}$ The difference in age and ethnicity between these studies may explain this controversy.

The present study limitation had some limitation, Firstly total testosterone was the only androgen that was measured in our study and other androgens such as androstenedione and dehydroepiandrosterone sulfate or free testosterone do not be analyzed because of the limitation of research funding. Secondly absence of assay of pituitary gondotrophins which have central role in pathogenesis of the disease. Thirdly exclusion of postmenopausal syndrome and congenital adrenal hyperplasia on clinical base only.

\section{CONCLUSION}

In conclusion VAI was an independent significant predictor for metabolic syndrome in patients with PCOS and a good marker of cardiometabolic risk in PCOS patients. In addition, VAI was a significant predictor of annual menusteral cycle but not testosterone levels or polycystic ovarian morphology in PCOS.

This study confirms the value of VAI in identification of patient with risk for metabolic syndrome and cardiometabolic risk in PCOS patients, but not found a role for VAI in diagnosis of PCOS.

\section{ACKNOWLEDGMENTS}

The Authors would like to thank to all for their help and support that was given to us.

Funding: No funding sources Conflict of interest: None declared

Ethical approval: The study was approved by the Institutional Ethics Committee

\section{REFERENCES}

1. Joham AE, Teede HJ, Ranasinha S, Zoungas S, Boyle J. Prevalence of infertility and use of fertility treatment in women with polycystic ovary syndrome: data from a large community-based cohort study. Journal of Women's Health. 2015;24(4):299-307. 
2. Zuo T, Zhu M, Xu W. Roles of oxidative stress in polycystic ovary syndrome and cancers. Oxid Med Cell Longev. 2016;2016:8589318.

3. Rotterdam ESHRE/ASRM-Sponsored PCOS consensus workshop group. Revised 2003 consensus on diagnostic criteria and long-term health risks related to polycystic ovary syndrome (PCOS). Human Reproduction. 2004;19(1):41-7.

4. Amato MC, Giordano C, Galia M, Criscimanna A, Vitabile S, Midiri M, et al. Visceral adiposity index: a reliable indicator of visceral fat function associated with cardiometabolic risk. Diabetes Care. 2010;33:920-2.

5. Amato MC, Giordano C, Pitrone M, Galluzzo A. Cut-off points of the visceral adiposity index (VAI) identifying a visceral adipose dysfunction associated with cardiometabolic risk in a Caucasian Sicilian population. Lipids Health Dis. 2011;10:183.

6. Zhang X, Shu XO, Li H, Yang G, Xiang YB. Visceral adiposity and risk of coronary heart disease in relatively lean Chinese adults. Int $\mathrm{J}$ Cardiol. 2013;168:2141-5.

7. Elisha B, Messier V, Karelis A, Coderre L, Bernard S, Prud'homme D, et al. The visceral adiposity index: relationship with cardiometabolic risk factors in obese and overweight postmenopausal women - a MONET group study. Appl Physiol Nutr Metab. 2013;38:892-9.

8. Petta S, Amato MC, Di Marco V, Cammà C, Pizzolanti G. Visceral adiposity index is associated with significant fibrosis in patients with nonalcoholic fatty liver disease. Aliment Pharmacol Ther. 2012;35:238-47.

9. Ciresi A, Amato MC, Pizzolanti G, Giordano Galluzzo C. Visceral adiposity index is associated with insulin sensitivity and adipocytokine levels in newly diagnosed acromegalic patients. J Clin Endocrinol Metab. 2012;97:2907-15.

10. Amato MC, Verghi M, Galluzzo A, Giordano C. The oligomenorrhoic phenotypes of polycystic ovary syndrome are characterized by a high visceral adiposity index: a likely condition of cardiometabolic risk. Hum Reprod. 2011;26:1486-94.

11. Bil E, Dilbaz B, Cirik DA, Ozelci R, Ozkaya E, Dilbaz S. Metabolic syndrome and metabolic risk profile according to polycystic ovary syndrome phenotype. J Obstet Gynaecol Res. 2016;42(7):83743.

12. Amato MC, Guarnotta V, Forti D, Donatelli M, Dolcimascolo S, Giordano C. etabolically healthy polycystic ovary syndrome (MH-PCOS) and metabolically unhealthy polycystic ovary syndrome (MU-PCOS): a comparative analysis of four simple methods useful for metabolic assessment. Hum Reprod. 2013;28(7):1919-28.

13. Oh JY, Sung YA, Lee HJ. The visceral adiposity index as a predictor of insulin resistance in young women with polycystic ovary syndrome. Obesity (Silver Spring). 2013;21(8):1690-4.
14. Zheng SH, Li XL. Visceral adiposity index as a predictor of clinical severity and therapeutic outcome of PCOS. Gynecol Endocrinol. 2016;32(3):177-83.

15. Amato MC, Magistro A, Gambino G, Vesco R, Giordano C. Visceral adiposity index and DHEAS are useful markers of diabetes risk in women with polycystic ovary syndrome. Eur J Endocrinol. 2015;172(1):79-88.

16. Jee-Young Oh, Yeon-Ah Sung and Hye Jin Lee. The visceral adiposity index as a predictor of insulin resistance in young women with polycystic ovary syndrome. Obesity. 2013;21:1690-4.

17. Androulakis II, Kandaraki E, Christakou C, Karachalios A, Marinakis E, Paterakis T, et al. Visceral adiposity index (VAI) is related to the severity of anovulation and other clinical features in women with polycystic ovary syndrome. Clin Endocrinol (Oxf). 2014;81(3):426-31.

18. Nestler JE, Powers LP, Matt DW, Steingold KA, Plymate SR, Rittmaster RS, et al. A direct effect of hyperinsulinemia on serum sex hormone-binding globulin levels in obese women with the polycystic ovary syndrome. J Clin Endocrinol Metab. 1991;72(1):83-9.

19. Ferriman D, Gallwey JD. Clinical assessment of body hair growth in women. Journal of Clinical Endocrinology and Metabolism. 1961;21:1440-7.

20. The Rotterdam ESHRE/ASRM-sponsored PCOS consensus workshop group. Revised 2003 consensus on diagnostic criteria and longterm health risks related to polycystic ovary syndrome (PCOS). Hum Reprod. 2004;19:41-7.

21. Wild RA, Carmina E, Diamanti-Kandarakis E. Assessment of cardiovascular risk and prevention of cardiovascular disease in women with the polycystic ovary syndrome: a consensus statement by the androgen excess and polycystic ovary syndrome (AE-PCOS) society. Journal of Clinical Endocrinology and Metabolism. 2010;95:2038-49.

22. Tosi F, Di Sarra D, Kaufman JM, Bonin C, Moretta $\mathrm{R}$, Bonora $\mathrm{E}$, et al. Total body fat and central fat mass independently predict insulin resistance but not hyperandrogenemia in women with polycystic ovary syndrome. J Clin Endocrinol Metab. 2015;100(2):661-9.

23. Diamanti-Kandarakis E, Dunaif A. Insulin resistance and the polycystic ovary syndrome revisited: an update on mechanisms and implications. Endocrine Reviews. 2012;3:981-1030.

24. Fulghesu AM, Angioni S, Belosi C, Apa R, Selvaggi L, Ciampelli M, et al. Pituitary-ovarian response to the gonadotrophin-releasing hormone-agonist test in anovulatory patients with polycystic ovary syndrome: predictive role of ovarian stroma. Clin Endocrinol (Oxf). 2006;65(3):396-401.

25. Kassi EN, Diamanti-Kandarakis E, Papavassiliou AG. Correlates between hyperinsulinism and hyperandrogenemia? Fertility and Sterility. 2008;89:1033-4. 
26. Panidis D, Tziomalos K, Papadakis E, Chatzis $P$, Kandaraki EA. Associations of menstrual cycle irregularities with age, obesity and phenotype in patients with polycystic ovary syndrome. Hormones (Athens). 2015;14(3):431-7.

27. Pasquali R, Pelusi C, Genghini S, Cacciari M, Gamineri A. Obesity and reproductive disorders in women. Hum Reprod Update. 2003;9:359-72.

28. Kirschner MA, Samojlik E, Drejka M, Szmal E, Schneider G, Ertel N. Androgen-estrogen metabolism in women with upper body versus lower body obesity. J Clin Endocrinol Metab. 1990;70(2):473-9.

29. Castillo-Martinez L, Lopez-Alvarenga JC, Villa AR, Gonzalez- Barranco J. Menstrual cycle length disorder in 18 - to 40 -y-old obese women. Nutrition. 2003;19:317-20.

30. Lambert-Messerlian G, Roberts MB, Urlacher SS, Ah-Ching J, Viali S, et al. First assessment of menstrual cycle function and reproductive endocrine status in Samoan women. Hum Reprod. 2011;26(9):2518-24.

31. Azziz R, Carmina E, DeWailly D. Position statement: criteria for defining polycystic ovary syndrome as a predominantly hyperandrogenic syndrome: an Androgen Excess Society guideline. J Clin Endocrinol Metab. 2006;91:4237-45.

32. Sirmans SM, Pate KA. Epidemiology, diagnosis, and management of polycystic ovary syndrome. Clinical Epidemiology. 2014;6:1-13.
33. Allsworth JE, Clarke J, Peipert JF, Hebert MR, CRNP AC, Boardman LA. The influence of stress on the menstrual cycle among newly incarcerated women. Women's health issues: official publication of the Jacobs Institute of Women's Health. 2007;17(4):202-9.

34. Bil E, Dilbaz B, Cirik DA, Ozelci R, Ozkaya E, Dilbaz S. Metabolic syndrome and metabolic risk profile according to polycystic ovary syndrome phenotype. J Obstet Gynaecol Res. 2016;42(7):83743.

35. Porter MB. Polycystic ovary syndrome: the controversy of diagnosis by ultrasound. Semin Reprod Med. 2008;26(3):241-51.

36. Dunaif A. Perspectives in polycystic ovary syndrome: from hair to eternity. J Clin Endocrinol Metab. 2016;101(3):759-68.

37. Techatraisak K, Wongmeerit K, Dangrat C, Wongwananuruk T, Indhavivadhana S. Measures of body adiposity and visceral adiposity index as predictors of metabolic syndrome among Thai women with PCOS. Gynecol Endocrinol. 2016;32(4):276-80.

38. Tehrani FR, Rashidi H, Khomami MB, Tohidi M, Azizi F. The prevalence of metabolic disorders in various phenotypes of polycystic ovary syndrome: a community based study in Southwest of Iran. Reprod Biol Endocrinol. 2014;12:89.

Cite this article as: Aboelnaga MM, Elshahawy $\mathrm{H}$. Visceral adiposity index correlation with Rotterdam criteria in patients with polycystic ovary syndrome. Int J Reprod Contracept Obstet Gynecol 2016;5:2553-60. 